\title{
CHARACTERIZATIONS OF NORMAL QUINTIC $K$-3 SURFACES
}

\author{
JIN-GEN YANG
}

\begin{abstract}
If a normal quintic surface is birational to a $K-3$ surface then it must contain from one to three triple points as its only essential singularities. A $K-3$ surface is the minimal model of a normal quintic surface with only one triple point if and only if it contains a nonsingular curve of genus two and a nonsingular rational curve crossing each other transversally. The minimal models of normal quintic $K-3$ surfaces with several triple points can also be characterized by the existence of some special divisors.
\end{abstract}

\section{INTRODUCTION}

Let $\mathbf{C}$ be the complex number field. A complete surface $S$ over $\mathbf{C}$ is a $K-3$ surface if the canonical divisor of $S$ is zero and $H^{1}(S)=0$. One of the simplest examples is a smooth quartic surface in $\mathbf{P}^{3}$. It was shown in [YJG] that some singular quintic surfaces are birational to $K-3$ surfaces. The aim of this paper is to find necessary and sufficient conditions for a $K-3$ surface to be birational to a normal quintic surface. The main results are

Theorem 1. A normal quintic surface in $\mathbf{P}^{3}$ is birational to a $K-3$ surface only if all its essential singularities are triple points.

Theorem 2. $A K-3$ surface $S$ is the minimal model of a normal quintic surface with one triple point as its only essential singularity if and only if there are two nonsingular curves $D$ and $B$ on $S$ with genus 2 and 0 respectively such that $D B=1$.

Theorem 3. $A K-3$ surface $S$ is the minimal model of a normal quintic surface with two triple points as its only essential singularities if and only if $S$ has one of the divisors listed in Figure 1.

(The solid dots are nonsingular elliptic curves. The hollow dots are nonsingular rational curves.)

Theorem 4. $A K-3$ surface $S$ is the minimal model of a normal quintic surface with more than two triple points if and only if there are three nonsingular elliptic curves $C_{1}, C_{2}$ and $C_{3}$ on $S$ with $C_{i} C_{j}=2$ for $1 \leq i<j \leq 3$.

A generic line passing through a triple point of a quintic surface meets the quintic surface at two other points besides the triple point. So it is natural to

Received by the editors January 29, 1988.

1980 Mathematics Subject Classification (1985 Revision). Primary 14J20; Secondary $14 J 17$. 


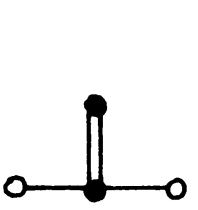

(a)

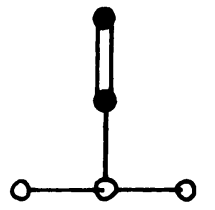

(b)

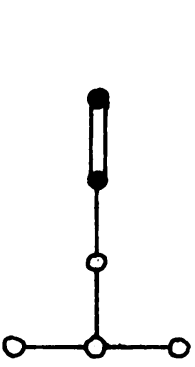

(c)

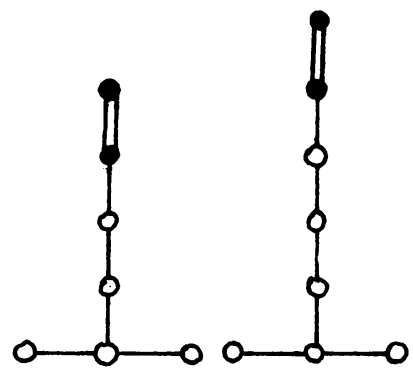

(d)

(e)

FIGURE 1

study the double cover of a normal quintic $K-3$ surface over a plane. In the last section some descriptions of the branch loci of such double coverings are given.

\section{Preliminaries}

In this section we briefly mention some standard notions concerning isolated singularities of surfaces. For details see [Art1, Art2, Lauf and Yau].

Let $p$ be an isolated singularity on a surface $V$ and let $\pi: M \rightarrow V$ be the minimal resolution of $p$. The number $h=\operatorname{dim}_{C} H^{0}\left(V, R^{1} \pi_{*}\left(O_{M}\right)\right)$ is the geometric genus of $p$. It is well known that

$\chi(V)=\chi(M)+h$

where $\chi(V)$ denotes the holomorphic Euler characteristic of $V$.

The set $A=\pi^{-1}(p)$ is called the exceptional set of $p$. Let $A=\bigcup A_{i}, 1 \leq$ $i \leq n$, be the decomposition of $A$ into irreducible components.

(Remark. If $p$ is a smooth point on a surface $V$ and let $f: X \rightarrow V$ be a birational morphism, then $f^{-1}(p)$ is also called the exceptional set of $p$ on $X$.)

A cycle $D$ on $A$ is an integral combination of the $A_{i}$ 's. There is a natural partial ordering, denoted by $<$, among cycles. For any closed subvariety $B$ of pure dimension 1 of $A$, there is a unique cycle $Z_{B}$ satisfying

(i) $\operatorname{Supp}\left(Z_{B}\right)=B$

(ii) $A_{i} Z_{B} \leq 0$ for all $A_{i} \leq B$;

(iii) $Z_{B}$ is minimal with respect to these two properties.

Such a cycle is called a fundamental cycle. In particular, $Z_{A}$ is the fundamental cycle of the singularity $p$, denoted by $Z$.

If $\chi(Z)=0$ then $p$ is called a weakly elliptic point. For any weakly elliptic point $p$, there is a unique cycle $E \leq Z$ such that $\chi(E)=0$ and $\chi(D)>0$ for all $0<D<E$. This $E$ is called the minimally elliptic cycle of $p$. If the fundamental cycle $Z$ itself is the minimally elliptic cycle then $p$ is called a minimally elliptic point. A singularity is called essential if it is not a rational double point. 


\section{NORMAL QUINTIC $K-3$ SURFACES WITH ONE TRIPLE POINT}

Throughout this paper a quintic $K-3$ surface will mean either a singular quintic surface in $\mathbf{P}^{3}$ which is birational to a $K-3$ surface or its birational model.

Let $S_{0}$ be a normal quintic surface and let $S$ be its minimal resolution. Since the divisor $S_{0}+K_{\mathrm{P}^{3}}$ in $\mathbf{P}^{3}$ is linearly equivalent to a hyperplane, an effective canonical divisor of $S$, if exists, is cut out by a hyperplane $H_{0}$ passing through all essential singularities of $S_{0}$. Let $C_{0}$ be the intersection of $S_{0}$ and $H$. If $S$ is birational to a $K-3$ surface, then the canonical divisor of $S$ is a collection of exceptional divisors of first kind. Hence all components of the proper transform of $C_{0}$ in $S$ must be exceptional curves of first kind. This indicates that there are not many quintic $K-3$ surfaces. In particular, if $S$ is already a minimal surface then $S$ cannot be a $K-3$ surface.

Lemma 2.1. A normal quintic surface with essential singularities, among which one is a double point, cannot be $K-3$.

Proof. Let $S_{0}$ be a normal quintic surface and let $p$ be an essential double point on $S_{0}$. Let $\varphi: T \rightarrow \mathbf{P}^{3}$ be the blowing-up of $\mathbf{P}^{3}$ at the point $p$ and let $E$ be the exceptional plane. Let $S$ be the proper transform of $S_{0}$. The canonical divisor $K_{T}$ of $T$ is $\varphi^{*}\left(K_{\mathbf{p}^{3}}\right)+2 E$ and the divisor $S$ is linearly equivalent to $\varphi^{*}\left(S_{0}\right)-2 E$. Thus $K_{T}+S$ is linearly equivalent to $\varphi^{*}(H)$ where $H$ is a hyperplane in $\mathbf{P}^{3}$.

Suppose that $S$ is birational to a $K-3$ surface. Then the canonical divisor of the minimal resolution of $S_{0}$ is cut out by a hyperplane $H_{0}$ passing through the point $p$. On $T$ the divisor $\varphi^{*}\left(H_{0}\right)$ is the union of $E$ and the proper transform of $H_{0}$. Let $S^{\prime}$ be the minimal resolution of $S$. Since $S$ has at most double points or double curves on $E$, the canonical divisor of $S^{\prime}$ contains the exceptional set $A$ of the double point $p$. Since $S^{\prime}$ is birational to a $K-3$ surface, the divisor $A$ is part of the exceptional set of a smooth point, which contradicts the assumption that $p$ is an essential singularity. Therefore $S_{0}$ cannot be $K-3$. Q.E.D.

Proof of Theorem 1. Let $S_{0}$ be a normal quintic surface. If $S_{0}$ has a 5-tuple point, then $S_{0}$ is a cone which is birational to a ruled surface. If $S_{0}$ has a 4-tuple point, then the projection from the 4-tuple point gives a birational map from $S_{0}$ to a rational surface. Lemma 2.1 says that $S_{0}$ is not $K-3$ if $S_{0}$ has essential double point. The conclusion follows immediately. Q.E.D.

Let $S_{0}$ be a quintic surface with a triple point $p$. We may assume that the equation of $S_{0}$ is

$$
f_{3}(x, y, z)+f_{3}(x, y, z)+f_{5}(x, y, z)=0
$$

where $f_{i}(x, y, z)$ is a homogeneous polynomial of degree $i$. 
Let $C$ be the plane cubic curve defined by the equation $f_{3}(x, y, z)=0$. Then the triple point $p$ has the following types in terms of the cubic curve $C$ :

(i) $C$ is reduced with at most ordinary double points (i.e., the rational double points of type $A_{1}$ );

(ii) $C$ is the union of a line and a conic tangent to each other;

(iii) $C$ is the union of three concurrent lines;

(iv) $C$ is the union of a line and a double line;

(v) $C$ is a triple line.

For details, see [YJG, §4].

Lemma 2.2. An isolated triple point of type (i) on a quintic surface is a minimally elliptic singularity.

Proof. See [YJG, pp. 445-446].

Lemma 2.3. Let $p$ be an isolated triple point of type (ii) on a quintic surface, then either $p$ is minimally elliptic or $p$ has an infinitely near essential double point.

Proof. [YJG, p. 446(v)].

Lemma 2.4. Let $S_{0}$ be a normal quintic surface with a triple point $p$ of type (ii) which is not minimally elliptic. Then $S_{0}$ is not $K-3$.

Proof. Let $\pi: T \rightarrow \mathbf{P}^{3}$ be the blowing-up of $\mathbf{P}^{3}$ at the point $p$ and let $E$ be the exceptional plane. Let $S$ be the proper transform of $S_{0}$. The canonical divisor $K_{T}$ of $T$ is $\pi^{*}\left(K_{\mathrm{P}^{3}}\right)+2 E$ and the divisor $S$ is linearly equivalent to $\pi^{*}\left(S_{0}\right)-3 E$. Thus $K_{T}+S$ is linearly equivalent to $\pi^{*}\left(H_{0}\right)-E$ where $H_{0}$ is a hyperplane in $\mathbf{P}^{3}$. So $K_{T}+S$ is linearly equivalent to the proper transform $H$ of $H_{0}$ in $T$. Let $S^{\prime}$ be the minimal resolution on $S$. Then the canonical divisor of $S^{\prime}$ is cut out by the plane $H_{0}$ in $\mathbf{P}^{3}$ whose proper transform $H$ passes through the essential double point of $S$. Then following the same argument as in the proof of Lemma 2.1 one sees that $S$ cannot be birational to a $K-3$ surface. Q.E.D.

Lemma 2.5. Let $S_{0}$ be a quintic surface with a triple point as its only essential singularity. If $S_{0}$ is $K-3$ then the triple point must have type (iv) or (v). Furthermore the blowing-up of $S_{0}$ at the triple point is not a normal surface.

Proof. If the triple point is a minimally elliptic point, then $S$ is birational to a surface of general type by computing the invariants. Hence Lemmas 2.2-2.4 imply that the triple point cannot have type (i) or (ii). If $p$ is of type (iii) then it was shown in [YJG, p. 446] that $S_{0}$ is either of general type or an elliptic surface with Kodaira dimension 1.

Hence the triple point must have type (iv) or (v). Let $S$ be the blowing-up of $S_{0}$ at the triple point. If $S$ is normal, then the Kodaira dimension of $S$ is either 1 or 2 [YJG, pp. 446-447]. Q.E.D. 
Lemma 2.6. Let $S$ be the minimal resolution of a quintic $K-3$ surface $S_{0}$. If there are five disjoint exceptional curves on first kind of $S$ then $S_{0}$ is normal.

Proof. The canonical divisor of $S$ is cut out by a hyperplane $H$ in $\mathbf{P}^{3}$. Suppose $S_{0}$ were not normal. Then $H \cap S_{0}$ would not be reduced, whence it would have less than five irreducible components. This implies that $S$ would have less than five disjoint exceptional divisors of first kind. Q.E.D.

Proof of Theorem 2. Assume that $S_{0}$ is birational to a $K-3$ surface. Let $T$ be the blowing-up of $\mathbf{P}^{3}$ and let $E$ be the exceptional plane. Let $S$ be the proper transform of $S_{0}$ in $T$. Because of Lemma 2.5 we may assume that $S_{0}$ has the equation

$$
y^{2} z+y f(x, y, z)+g(x, y, z)=0
$$

or

$$
y^{3}+y f(x, y, z)+g(x, y, z)=0,
$$

where $f(x, y, z)$ and $g(x, y, z)$ are homogeneous polynomials in $x, y, z$ with degrees 3 and 5 respectively. Let $H_{0}$ be a generic plane in $\mathbf{P}^{3}$ passing through the triple point $p$. Bertini's Theorem implies that the intersection $C_{0}$ of $H_{0}$ and $S_{0}$ is an irreducible quintic curve with $p$ as its only singularity. The equations (2) and (3) imply that $C_{0}$ has a triple point with an infinitely near double point at $p$. Therefore $C_{0}$ has geometric genus 2 .

Assume that the equation for $S_{0}$ is (2). Then $E \cap S$ is the union of a line $L_{1}$ and a double line $L_{2}$. Let $H$ be the proper transform of $H_{0}$ in $T$. Since $H_{0}$ is in general position, $H$ meets $L_{1}$ and $L_{2}$ at two distinct points $s_{1}$ and $s_{2}$ respectively. Let $C$ be the proper transform of $C_{0}$ in $S$. Then $C$ is smooth at $s_{1}$ and $C$ has a double point at $s_{2}$. Note that $S$ is singular along $L_{2}$. The blowing-up of $T$ along $L_{2}$ will normalize $S$ and $C$ at the same time. Let $S^{\prime}$ be the minimal resolution of $S$. Then the proper transform $C^{\prime}$ of $C$ in $S^{\prime}$ is a nonsingular curve of genus 2 and the proper transform $L_{1}^{\prime}$ of $L_{1}$ in $S^{\prime}$ intersects $C^{\prime}$ transversally, because $S$ is smooth at the point $S_{1}$ thanks to the general position of $H$. On the other hand the canonical divisor, which is a collection of exceptional divisors of first kind, is cut out by the plane $y=0$ in $\mathbf{P}^{3}$. So the exceptional divisors of first kind on $S^{\prime}$ do not meet $C^{\prime}$. Let $D$ and $B$ be the image of $C^{\prime}$ and $L_{1}^{\prime}$ in the minimal model of $S^{\prime}$. Then $D B=1$ and $D^{2}=2, B^{2}=-2$ by the adjunction formula.

Next we assume that the equation of $S_{0}$ in (3). Then $E \cap S$ is a triple line $L$. Let $H$ be the proper transform of $H_{0}$. Let $C$ be the proper transform of $C_{0}$ in $S$. Then $C$ has a double point at $C \cap L$. Let $T^{*}$ be blowing-up of $T$ along $L$ and let $F$ be the exceptional divisor. Let $S^{*}$ be the proper transform of $S$. The equation (3) reveals that the intersection of $F$ and the proper transform of $E$ in $T^{*}$ is a rational curve $L^{*}$, which lies in $S^{*}$. The proper transform $C^{*}$ of $C$ is a nonsingular curve meeting $L^{*}$ transversally. Since $H_{0}$ is in general position, $S^{*}$ is smooth at $C^{*} \cap L^{*}$ and there is no exceptional divisor of first 
kind passing through $C^{*} \cap L^{*}$. Let $D$ and $B$ be the image of $C^{*}$ and $L^{*}$ in the minimal model of $S^{*}$ respectively. Then $D B=1, D^{2}=2$ and $B^{2}=-2$.

Conversely let $S$ be a $K-3$ surface such that there are two nonsingular curves $D$ and $B$ with genera 2 and 0 respectively on $S$ such that $D B=1$. We want to show that $S$ is the minimal model of a quintic $K-3$ surface.

The adjunction formula implies that $D^{2}=2$ and $B^{2}=-2$. Let $k$ be the canonical divisor of the curve $D$. Then $\operatorname{deg}(k)=2$. Let $p$ be the intersection point of $D$ and $B$. Then $h^{0}(D, O(2 k+p))=h^{0}(D, O(2 k))+1=4$ by the Riemann-Roch theorem. Hence a general member of the linear system $|2 k+p|$ consists of five distinct points $p_{1}, p_{2}, p_{3}, p_{4}, p_{5}$ of which none is the point $p$.

Lemma 2.7. Every pair of points among $p_{1}, p_{2}, p_{3}, p_{4}, p_{5}$ is not linearly equivalent to the canonical divisor $k$.

Proof. If $p_{1}+p_{2}$ were linearly equivalent to $k$, then $p_{3}+p_{4}+p_{5}$ would be linearly equivalent to $k+p$. Since $h^{0}(D, O(k))=h^{0}(D, O(k+p))=2$, one of $p_{3}, p_{4}, p_{5}$ would be $p$. This would contradict our choice of $p_{1}, \ldots, p_{5}$. Q.E.D.

Let $S^{\prime}$ be the blowing-up of $S$ at these five points and let $E_{1}, \ldots, E_{5}$ be the exceptional divisors. Let $D^{\prime}$ and $B^{\prime}$ be the proper transforms of $D$ and $B$ respectively. Since $K_{S}=0, h^{1}\left(D, O_{D}(D)\right)=h^{0}\left(D, O_{D}\right)=1$ by the adjunction formula. The short exact sequence

$$
0 \rightarrow O_{S} \rightarrow O_{S}(D) \rightarrow O_{D}(D) \rightarrow 0
$$

implies that

$$
h^{o}(S, O(D))=3 \quad \text { and } \quad h^{1}(S, O(D))=0 .
$$

Hence $h^{0}\left(S^{\prime}, O\left(D^{\prime}+E_{1}+\cdots+E_{5}\right)\right)=3$ and $h^{1}\left(S^{\prime}, O\left(D^{\prime}+E_{1}+\cdots+E_{5}\right)\right)=0$. The short exact sequence

$$
0 \rightarrow O_{S^{\prime}}\left(D^{\prime}+E_{1}+\cdots+E_{5}\right) \rightarrow O_{S^{\prime}}\left(D^{\prime}+B^{\prime}+E_{1}+\cdots+E_{5}\right) \rightarrow O_{B^{\prime}}(-1) \rightarrow 0
$$

implies that

$h^{0}\left(S^{\prime}, O\left(D^{\prime}+B^{\prime}+E_{1}+\cdots+E_{5}\right)\right)=3, \quad h^{1}\left(S^{\prime}, O\left(D^{\prime}+B^{\prime}+E_{1}+\cdots+E_{5}\right)\right)=0$.

Let $H=2 D^{\prime}+B^{\prime}+E_{1}+\cdots+E_{5}$. Since $P_{1}+P_{2}+P_{3}+P_{4}+P_{5}$ is linearly equivalent to $2 k+p$ on $D$, the restriction of the divisor $H$ on $D^{\prime}$ is linearly equivalent to 0 on $D^{\prime}$. Hence the short exact sequence

$$
0 \rightarrow O_{S^{\prime}}\left(D^{\prime}+B^{\prime}+E_{1}+\cdots+E_{5}\right) \rightarrow O_{S^{\prime}}(H) \rightarrow O_{D^{\prime}} \rightarrow 0
$$

implies that $h^{0}\left(S^{\prime}, O(H)\right)=4$. Next we want to show that this linear system has neither fixed components nor base points. Since $h^{0}\left(S, O\left(H-D^{\prime}\right)\right)=3, D^{\prime}$ is not a fixed component of $|H|$. Since $H D^{\prime}=0$, there are no base points on $D^{\prime}$. Let $H_{1}$ be a member of $|H|$ which does not contain $D^{\prime}$. Since $H D^{\prime}=0$, $H_{1}$ must not contain $B^{\prime}$ or any of $E_{i}$. Therefore $|H|$ has no fixed components. A result of Saint-Donat says that on a $K-3$ surface any linear system without 
fixed components has no base points [Sai]. Thus the linear system $|D|$ on $S$ has no base points. Hence there is an effective divisor $D_{1}$ on $S^{\prime}$ which is linearly equivalent to $D^{\prime}+E_{1}+\cdots+E_{5}$ and does not meet $E_{1}$. The divisor $D^{\prime}+D_{1}+B^{\prime}$ is linearly equivalent to $H$ which meets $E_{1}$ at $E_{1} \cap D^{\prime}$. Since $H_{1}$ does not meet $D^{\prime}, H_{1}$ and $D^{\prime}+D_{1}+B^{\prime}$ have no common points on $E_{1}$. Hence the linear system $|H|$ has no base points on $E_{1}$. For the same reason it has no base points on all $E_{i}$. Therefore the linear system $|H|$ is base point free. The linear system $|H|$ defines a morphism $\varphi$ from $S^{\prime}$ to $\mathrm{P}^{3}$. Since $H E_{i}=1$, the images of $E_{1}, \ldots, E_{5}$ are lines. Lemma 2.7 implies that for every pair $1 \leq i<j \leq 5$ there is a member $H^{*}$ in $|H|$ which contains $E_{i}$ but not $E_{j}$. Hence the images of $E_{1}, \ldots, E_{5}$ are distinct. Since $H^{2}=5$, the image of $S^{\prime}$ under $\varphi$ is a quintic surface. Hence $\varphi$ is a birational morphism. Since the images of $E_{1}, \ldots, E_{5}$ are lines, the minimal resolution of the image of $S^{\prime}$ has five disjoint exceptional curves of first kind. By Lemma 2.6, the image of $S^{\prime}$ is normal. Suppose that $F$ is a curve on $S^{\prime}$ disjoint from $H$ whose image in $\mathbf{P}^{3}$ is a point. Then the algebraic index theorem implies that $F^{2}<0$. Since $F K_{S^{\prime}}=0$, the adjunction formula implies that $\chi(F)>0$. Hence the image of $F$ is a rational double point. Therefore the birational image of $S^{\prime}$ in $\mathbf{P}^{3}$ is a normal quintic surface with a triple point as its only essential singularity. Q.E.D.

\section{NORMAL QUINTIC SURFACES WITH SEVERAL TRIPLE POINTS}

In this section we discuss the normal quintic surfaces with more than one triple points.

Lemma 3.1. Let $S_{0}$ be a normal quintic surface with more than one triple points. Assume that one triple point $p$ has type (iv) or (v) and the blowing-up of $S_{0}$ at $p$ is not a normal surface. Then $S_{0}$ is not $K$-3.

Proof. We may assume that $p$ has the equation (2) or (3). The canonical divisor of the minimal resolution of $S_{0}$ is cut out by the plane $y=0$. Let $q$ be another triple point on $S_{0}$. It suffices to show that $q$ is not on the plane $y=0$, because then the canonical divisor of the minimal model will be $-D$ where $D$ is the union of anticanonical divisors of all triple points other than $p$.

Suppose that $q$ were on the plane $y=0$. With a suitable linear transformation, we may assume that $q=(\infty, 0,0)$. That would imply that the exponent of $x$ in each term of (2) or (3) is less than or equal to 2, whence the surface $S_{0}$ is singular along the line $y=0, z=0$. This would contradict the assumption that $S_{0}$ is normal. Q.E.D.

Lemma 3.2. Let $p$ be a minimally elliptic triple point on a normal surface $S_{0}$ in $\mathbf{P}^{3}$ and let $H_{0}$ be a plane passing through $p$. Let $C_{1}, C_{2}$ and $C_{3}$ be three curves on the plane $H_{0}$ such that (i) all $C_{i}$ pass through $p$; (ii) all $C_{i}$ are smooth at $p$ and (iii) $C_{i}$ and $C_{j}$ intersect at $p$ transversally at $p$ for $i \neq j$. Let $S^{\prime}$ be the 
minimal resolution of $S_{0}$. Let $Z$ be the fundamental cycle of $p$. Let $C_{1}^{\prime}, C_{2}^{\prime}$ and $C_{3}^{\prime}$ be the proper transforms of $C_{1}, C_{2}$ and $C_{3}$ in $S^{\prime}$ respectively. Then $C_{i}^{\prime} Z=1$ for $i=1,2,3$.

Proof. Let $T$ be the blowing up of $\mathbf{P}^{3}$ at $p$ and let $E$ be the exceptional plane. Let $S$ be the proper transform of $S_{0}$. Then the curve $C=E \cap S$ is a plane cubic curve. Thus the intersection of the proper transform of $H_{0}$ and $C$ consist of three points $a, b, c$. Since the tangent directions of $C_{1}, C_{2}$ and $C_{3}$ at $p$ are distinct, the three points $a, b, c$ on $E$ must be distinct and $C$ must be smooth at these three points. Hence the proper transforms of $C_{1}, C_{2}$ and $C_{3}$ meet $C$ at $a, b$ and $c$ transversally. Since $p$ is a minimally elliptic point, there are at most rational double points for $S$ on $C$ and none of $a, b, c$ is a rational double point. The result follows immediately. Q.E.D.

Lemma 3.3. Let $S_{0}$ be a normal quintic $K$-3 surface with more than 2 triple points. Then $S_{0}$ has exactly 3 minimally elliptic triple points which are not collinear. The minimal model of $S_{0}$ contains three nonsingular elliptic curves $D_{1}, D_{2}$ and $D_{3}$ with $D_{i} D_{j}=2$ for all $i \neq j$.

Proof. Since each triple point has a positive geometric genus. The sum of the geometric genera of the triple points of $S_{0}$ must be 3. This implies that $S_{0}$ has exactly three triple points $p, q, r$ and all of them are minimally elliptic. Let $L_{p q}$ be the line passing through $p$ and $q$. Then $L_{p q}$ must be on $S_{0}$, otherwise the intersection number of $L_{p q}$ and $S_{0}$ would be greater than 5 , which is impossible. Let $H$ be a generic plane passing through $L_{p q}$. The intersection of $H$ and $S_{0}$ is the union of $L_{p q}$ and a quartic curve $Q$. Since $p$ and $q$ are triple points of the plane curve $L_{p q} \cup Q, L_{p q}$ meets $Q$ at $p$ and $q$ only. This implies that the triple point $r$ is not on $L_{p q}$. Let $L_{p r}$ and $L_{q r}$ be the lines passing through $p, r$ and $q, r$ respectively and let $H_{p q r}$ be the plane passing through $p, q$ and $r$. Then $H_{p q r} \cap S_{0}$ is the union of $L_{p q}, L_{p r}, L_{q r}$ and a conic $C$ which passes through $p, q$ and $r$.

Let $S^{\prime}$ be the minimal resolution of $S_{0}$. Let $Z_{p}, Z_{q}$ and $Z_{r}$ be the fundamental cycles of $p, q$ and $r$ and $S^{\prime}$ respectively. Let $L_{p q}^{\prime}, L_{p r}^{\prime}, L_{q r}^{\prime}$ and $C^{\prime}$ be the proper transforms of $L_{p q}, L_{p r}, L_{q r}$ and $C$ on $S^{\prime}$ respectively. By Lemma 3.2 $L_{p q}^{\prime} Z_{p}=L_{p r}^{\prime} Z_{p}=C^{\prime} Z_{p}=1$ and etc. Let $S^{\prime} \rightarrow S$ be the blowingdown of $L_{p q}^{\prime}, L_{p r}^{\prime}, L_{q r}^{\prime}$ and $C^{\prime}$. Then $S$ is a $K-3$ surface. Let $B_{1}, B_{2}$ and $B_{3}$ be the direct images of $Z_{p}, Z_{q}$ and $Z_{r}$ in $S$ respectively. They are all minimally elliptic cycles. The Riemann-Roch theorem implies that the linear system $\left|B_{i}\right|$ has dimension 1 for each $i$. Since $B_{i}$ is minimally elliptic, $\left|B_{i}\right|$ has no fixed components. Take a general member $D_{i}$ from each $\left|B_{i}\right|$. Then $D_{1}, D_{2}$ and $D_{3}$ are nonsingular elliptic curves on $S$ with $D_{i} D_{j}=2$ for all $i \neq j$. Q.E.D. 
Lemma 3.4. Let $S_{0}$ be a normal quintic $K-3$ surface with two triple points $p$ and $q$ as its only essential singularities. Then the minimal model of $S_{0}$ contains one of the divisors in Figure 1.

Proof. We may assume that the geometric genera of $p$ and $q$ are 2 and 1 respectively. By Lemmas 2.4 and $3.1 p$ is a triple point of type (iii), (iv) or (v) with an infinitely near triple point.

We may assume that the equation of $S_{0}$ has the form

$$
f_{3}(x, y, z)+f_{4}(x, y, z)+f_{5}(x, y, z)=0
$$

with $p=(0,0,0)$ and $q=(0,0, \infty)$. Here $f_{i}(x, y, z)$ are homogeneous polynomials of degree $i$ for $i=3,4,5$. Since $q$ is a triple point, the exponent of $z$ in each term of (4) is less than three. So $f_{3}(x, y, z)$ does not contain the monomial $z^{3}$. Either $x z^{2}$ or $y z^{2}$ must appear in $f_{3}(x, y, z)$, otherwise $S_{0}$ would be singular along the line $x=0, y=0$. Immediately we see that $p$ cannot have type $(\mathrm{v})$. Without loss of generality we may assume that $f_{3}(x, y, z)$ is $y z^{2}$ or $y z(y-z)$. Let $L$ be the line $y=0, z=0$. This is the line whose proper transform passes through the infinitely near triple point of $p$. Since $S_{0}$ is assumed to have an infinitely near triple point, neither $x^{4}$ nor $x^{5}$ appears in the equation (4). Hence the line $L$ is on $S_{0}$.

Let $H$ be a generic plane passing through the line $L$. Then $H \cap S_{0}$ is the union of $L$ and an irreducible quartic curve $Q$ with a double point plus an infinitely near double point at $p$. Thus $Q$ has geometric genus 1 . The proper transform $D$ of $Q$ in the minimal model $S^{*}$ of $S_{0}$ is a nonsingular elliptic curve.

Let $T$ be the blowing-up of $\mathbf{P}^{3}$ at the point $p$ and let $E$ be the exceptional plane. Let $S$ be the proper transform of $S_{0}$. The intersection $E \cap S$ is the union of three lines $L_{1}, L_{2}$ and $L_{3}$. One of them, say $L_{1}$, is on the proper transform of the plane $H_{0}$ in $\mathbf{P}^{3}$ passing through $L$ and $q$. The intersection point $s$ of $L_{1}, L_{2}$ and $L_{3}$ is a minimally elliptic triple point of $S$ and the proper transform of $Q$ meets $E$ at the point $s$ twice. Let $\pi: S^{\prime} \rightarrow S$ be the minimal resolution of $S$. The fundamental cycle of the triple point $s$ in $S^{\prime}$ is a minimally elliptic cycle $Z^{\prime}$, which meets the proper transform of $Q$ twice.

If the triple point $p$ is of type (iii), then $L_{2} \neq L_{3}$. Evidently the proper transforms of $Q, Z^{\prime}$ (which can be replaced by a generic member in its linear system), $L_{2}$ and $L_{3}$ in the minimal model of $S^{\prime}$ have the configuration (a) in Figure 1.

If the triple point $p$ has type (iv), then $L_{2}=L_{3}$. There are following cases:

(A) $S$ has two ordinary double points on $L_{2}$ away from $s$. Then the minimal model of $S^{\prime}$ contains a divisor (b) in Figure 1.

(B) $S$ has one double point $t$ on $L_{2}$ away from $s$ and $S$ has an infinitely near double point over the point $t$. That double point $t$ can be represented by one of the following three equations:

$$
z^{2}+x^{2}+x y^{2}=0, \quad z^{2}+x^{3}+x y^{2}=0, \quad z^{2}+x^{4}+x y^{2}=0 .
$$


Hence the minimal model of $S^{\prime}$ contains a divisor (c), (d) or (e) in Figure 1.

(C) $S$ has only one ordinary double point $t$ on $L_{2}$ away from $s$. Then $S$ has an infinitely near rational double point over the point $s$. Thus the divisor $Z^{\prime}$ contains a rational component $A_{i}$ intersecting the proper transform of $L_{2}$ transversally. Let $D$ be a general member of the linear system $\left|Z^{\prime}\right|$. Let $L^{\prime}$ be the rational exceptional curve of the double point $t$ and let $M$ be the proper transform of $L_{2}$ in $S^{\prime}$. Then $D, M, L^{\prime}$ and $A_{i}$ have the configuration in Figure 2, because $Z^{\prime} A_{i}=0$. Therefore the minimal model of $S^{\prime}$ contains a divisor (b) in Figure 1.

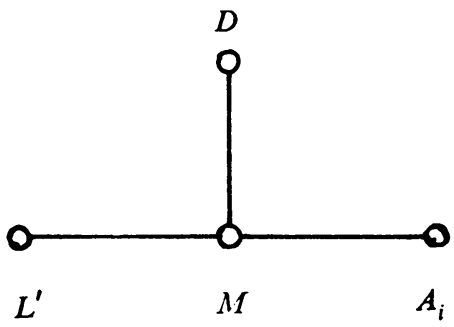

FIGURE 2

(D) $s$ is the only singularity of $S$ along the curve $L_{2}$. Then the cycle $Z^{\prime}$ contains a subcycle of type $A_{3}, D_{4}$ or $D_{5}$. following the same argument as in Case (C), one can see that the minimal model of $S^{\prime}$ contains a cycle (c), (d) or (e) in Figure 1. Q.E.D.

Proof of Theorem 4. The only part is a consequence of Lemma 3.3.

Suppose $S$ is a $K-3$ surface with three nonsingular elliptic curves $D_{1}, D_{2}$ and $D_{3}$ with $D_{i} D_{j}=2$ for $i \neq j$. We will show that $S$ is birational to a quintic surface with three triple points.

Obviously these three elliptic curves are in distinct linear systems. By proper choosing the representatives in these linear systems, we may assume that $D_{1}$, $D_{2}$ and $D_{3}$ have the configuration in Figure 3.

We obtain a divisor $H=L_{1}+L_{2}+L_{3}+Q+E_{1}+E_{2}+E_{3}$ on a surface $S^{\prime}$ as shown in Figure 4 by blowing-up the four intersection points in Figure 3.

Here $E_{1}, E_{2}, E_{3}$ are the proper transforms of $D_{1}, D_{2}$ and $D_{3}$ respectively and $L_{1}, L_{2}, L_{3}$ and $Q$ are the exceptional curves. The self-intersections are $L_{i}^{2}=Q^{2}=-1$ and $E_{i}^{2}=-2$ for $i=1,2,3$. One can show that $h^{0}\left(S^{\prime}, 0(H)\right)=4$ and that $|H|$ has neither fixed components nor base points. Since $H^{2}=5$, the complete linear system defines a birational morphism from $S^{\prime}$ to a quintic surface in $\mathbf{P}^{3}$. Let $D_{1}^{\prime}$ be a divisor on $S$ which is linearly equivalent to but not equal to $D_{1}$. Let $E_{1}^{\prime}$ be the pull-back of $D_{1}^{\prime}$ in $S^{\prime}$. Then 


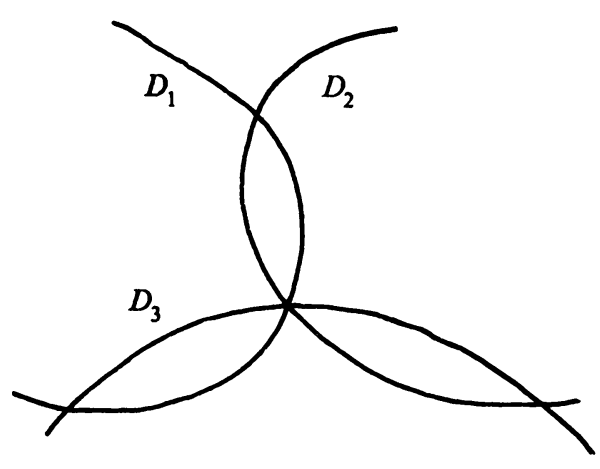

FIGURE 3

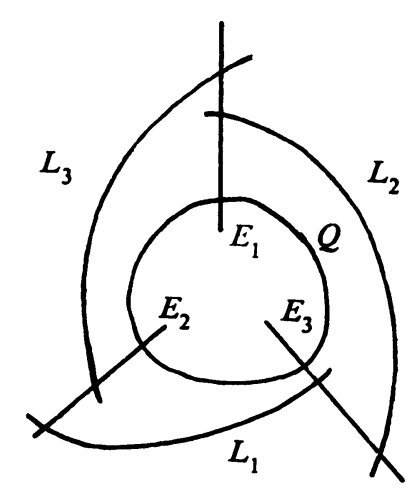

FIGURE 4

the divisor $E_{1}^{\prime}+E_{2}+E_{3}+L_{1}$ is linearly equivalent to $H$. Hence the image of $L_{1}$ in $S^{\prime}$ is different from those of $L_{2}, L_{3}$ and $Q$. Hence the image of the divisor $H$ is a reduced quintic curve, which consists of three lines and a conic. Therefore the quintic surface must be normal. Since $E_{i} H=0$ for $i=1,2,3$. The images of $E_{1}, E_{2}$ and $E_{3}$ are three isolated essential singularities. By Lemma 2.1, these must be triple points.

Proof of Theorem 3. The only part is a consequence of Lemma 3.4.

Assume that $S$ is a $K-3$ surface containing a divisor in Figure 1. One can use the same method to blow up some points to get a surface $S^{\prime}$ with a connected divisor $H$ satisfying $H^{2}=5, h^{0}\left(S^{\prime}, 0(H)\right)=4$ and $|H|$ has neither fixed components nor base points. For instance in the case (a) of Figure 1, we may assume that the divisor is $D_{1}+D_{2}+L+M$ as in Figure 5 .

Choose a general point $s$ on $D_{2}$. Blow up $S$ at $s$ and at the two intersection points of $D_{1}$ and $D_{2}$ to get a divisor in Figure 6. 


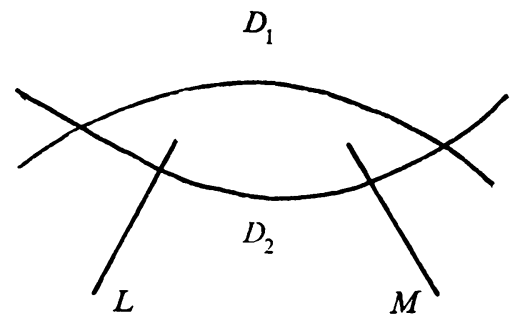

FIGURE 5

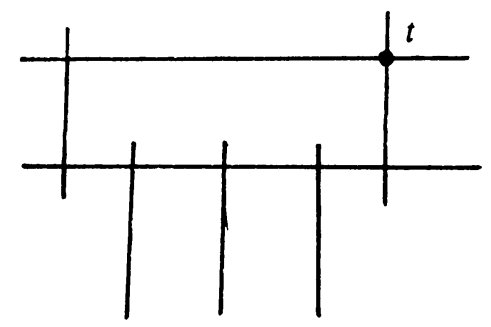

FIGURE 6

Then blow up the surface at the point $t$ to get a surface $S^{\prime}$ with a divisor $H=C_{1}+2 C_{2}+E_{1}+2 E_{2}+2 E_{3}+E_{4}+L_{1}+L_{2}$ as in Figure 7. Then one can check that the divisor $H$ satisfies all the conditions. It can be verified that $|H|$ defines a birational morphism from $S^{\prime}$ onto a normal quintic surface in $\mathbf{P}^{3}$. We leave the verifications of the other cases of Figure 1 to the readers. Q.E.D.

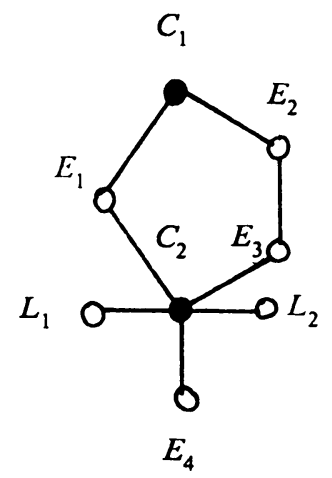

FIGURE 7 


\section{Characterizations by double planes}

Let $B$ be a reduced sextic curve on the plane $P^{2}$ without quadruple points and infinitely near triple points or worse singularities. Let $S$ be the double cover of $P^{2}$ with $B$ as the branch locus. Then $S$ is a $K-3$ surface (with possibly some rational double points). The following theorem identifies those sextic curves that will give rise to normal quintic $K-3$ surfaces with one triple point.

Theorem 4.1. $A K-3$ surface $S$ is the minimal model of a normal quintic $K-3$ surface with one triple point as its only essential singularity if and only if it is a double plane branched over a sextic curve $B$ without quadruple or infinitely near triple points and $B$ either has a tritangent line or contains a line.

Remark. Here a tritangent line is a line $L$ on $P^{2}$ such that all the intersection numbers $(L, B)_{p}$ are even for every point $p$.

Proof. According to Theorem 2, if $S$ is the minimal model of a normal quintic $K-3$ surface with one triple point, then there are nonsingular curves $D$ and $C$ with genera 2 and 0 respectively such that $D C=1$. The linear system $|D|$ is base point-free. It defines a double cover over $\mathbf{P}^{2}$ branched over a sextic curve $B$. Since $D C=1$, the image of $C$ is a line $L$ and the line $L$ either splits or is the branch locus. If $L$ splits, then $L$ is a tritangent line of $B$.

Conversely, if $S$ is a double cover branched over a sextic curve $B$ and if $L$ is a tritangent line to $B$, let $H$ be a line in general position. The inverse image of $H$ under the double cover is a nonsingular curve $D$ of genus 2. Let $L$ split into $C$ and $C^{\prime}$. Then $C$ is a rational curve and $D C=1$. Since $H$ is in general position, the surface $S$ is smooth at the point $D \cap C$. Hence Theorem 2 implies that the minimal model of $S$ is the minimal model of a normal quintic $K-3$ surface with one triple point as its only essential singularity. If the sextic curve $B$ contains a line $L$, then the inverse image of $L$ is a rational curve $C$ with $D C=1$. Once again the surface $S$ is the minimal model of a normal quintic $K-3$ surface with one triple point by Theorem 2. Q.E.D.

Some normal quintic $K-3$ surfaces with several triple points are also birational to sextic double planes, as can be seen by the following examples:

Examples. (1) Let $B$ be a plane sextic curve with three ordinary double points $p_{1}, p_{2}$ and $p_{3}$ as its only singularities. Let $S$ be the canonical resolution of the double cover of $\mathrm{P}^{2}$ branched over $B$. Let $L_{1}, L_{2}$ and $L_{3}$ be three generic lines on $\mathrm{P}^{2}$ passing through $p_{1}, p_{2}$ and $p_{3}$ respectively. Then $L_{i}$ meets $B$ at four other points besides $p_{i}$ and the intersections at these four points are transversal for each $i$. Hence the proper transforms of $L_{1}, L_{2}$ and $L_{3}$ in $S$ are three nonsingular elliptic curves with mutual intersection number 2 . Thus $S$ is birational to a normal quintic $K-3$ surface with three triple points by Theorem 4. 
(2) Let $B$ be a plane sextic curve with an ordinary double point $p_{1}$ and a double point $p_{2}$ which has an infinitely near double point. Let $S$ be the canonical resolution of the double cover of $\mathbf{P}^{2}$ branched over $B$. Let $L_{1}$ and $L_{2}$ be two generic lines on $\mathbf{P}^{2}$ passing through $p_{1}$ and $p_{2}$ respectively. Then the proper transforms $C_{1}, C_{2}$ of $L_{1}, L_{2}$ on $S$ are nonsingular elliptic curves with $C_{1} C_{2}=2$. Since $B$ has an infinitely near double point over $p_{2}$, there are two disjoint nonsingular rational curves $E_{1}$ and $E_{2}$ on $S$ meeting $C_{2}$ transversally. Thus $S$ has a divisor of (a) in Figure 1. Hence $S$ is birational to a normal quintic $K-3$ surface with two triple points.

Proposition 4.2. Any normal quintic $K-3$ surface with more than one triple points is birational to a double cover of $\mathbf{P}^{2}$ branched over an octic curve with two ordinary quadruple points such that the line passing through these two quadruple points is not a component of the branch locus.

Proof. Let $S$ be the minimal model of a normal quintic $K-3$ surface with more than one triple points. Then there are two nonsingular elliptic curves $C_{1}$ and $C_{2}$ with $C_{1} C_{2}=2$. We may assume that $C_{1}$ and $C_{2}$ intersect at two distinct points $p$ and $q$ without loss of generality. Let $S^{\prime}$ be the blowing up of $S$ at $p$ and $q$. Let $D_{1}$ and $D_{2}$ be the proper transforms of $C_{1}$ and $C_{2}$ respectively and let $E$ and $F$ be the two exceptional curves of first kind on $S^{\prime}$. Let $H=D_{1}+D_{2}+E+F$. It's easy to see that $h^{0}\left(S^{\prime}, 0(H)\right)=3$ and the linear system $|H|$ has neither fixed components nor base points. Since $H^{2}=2$ the linear system defines a double cover over $\mathbf{P}^{2}$. Since $H E=H F=1$ and $H D_{1}=H D_{2}=0$, the images of $E$ and $F$ are the same line $L$ on $\mathbf{P}^{2}$. Since $L$ splits in the double cover, $L$ must not be a component of the branch locus. The image of $D_{1}$ and $D_{2}$ are two points on $L$. Since both $D_{1}$ and $D_{2}$ are nonsingular elliptic curves, their images are ordinary quadruple points on the branch locus. Q.E.D.

Finally we give some examples of double octic planes which are birational to normal quintic $K-3$ surfaces.

Examples. (3) Let $C$ be a septic curve on $\mathbf{P}^{2}$ with an ordinary triple point $p$ and an ordinary quadruple point $q$ so that the line passing through $p$ and $q$ is not a component of $C$. Let $L$ be a generic line on $\mathbf{P}^{2}$ passing through the point $p$. Let $S$ be the canonical resolution of the double cover of $\mathbf{P}^{2}$ branched over the octic curve $B=C+L$. Then the line passing through $p$ and $q$ splits into two exceptional curves of first kind $E_{1}$ and $E_{2}$ on $S$. Let $D_{1}$ and $D_{2}$ be the inverse image of $p$ and $q$ in $S$. They are nonsingular elliptic curves. After blowing down $E_{1}$ and $E_{2}$, we get two nonsingular elliptic curves intersecting at two points. Since the proper transform of $L$ in $S$ is a nonsingular rational curve which connects to $D_{1}$ and four other nonsingular rational curves. In particular, $S$ contains a divisor (b) in Figure 1 . Hence $S$ is birational to a normal quintic $K-3$ surface with two triple points. 
(4) Let $B$ be a plane octic curve with two ordinary quadruple points $p, q$ and two ordinary double points $r, s$ as its only singularities. Assume that $p, r$ and $s$ are collinear and the line passing through $p$ and $q$ is not a component of $B$. Let $L$ be the line passing through $p$ and $q$ and let $M$ be the line passing through $p, r$ and $s$. The line $M$ is not a component of $B$, for otherwise $B$ would have more singularities. Then the line $M$ splits in the double cover. The minimal model of the double cover of $\mathbf{P}^{2}$ branched over $B$ contains a divisor (a) in Figure 1. Hence this surface is birational to a normal quintic $K-3$ surface with two triple points.

\section{REFERENCES}

[Art1] M. Artin, On isolated rational singularities of surfaces, Amer. J. Math. 88 (1966), 129-136. [Art2] __, Some numerical criteria for contractibility of curves on an algebraic surface, Amer. J. Math. 84 (1962), 485-496.

[Bvl] A. Beauville, Surfaces algébriques complexes, Asterisque 54, Soc. Math. France, Paris (1978)

[Hart] R. Hartshorne, Algebraic geometry, Springer-Verlag, Berlin and New York, 1977.

[Lauf] H. Laufer, On minimally elliptic singularities, Amer. J. Math. 99 (1977), 1257-1295.

[Sai] B. Saint-Donat, Projective models on K 3-surfaces, Amer. J. Math. 96 (1974), 602-639.

[Yau] S. S-T. Yau, On maximally elliptic singularities, Trans. Amer. Math. Soc. 257 (1980), 269329.

[YJG] Jin-Gen Yang, On quintic surfaces of general type, Trans. Amer. Math. Soc. 295 (1986), 431-473.

Institute of Mathematics, Fudan University, Shanghai, China 